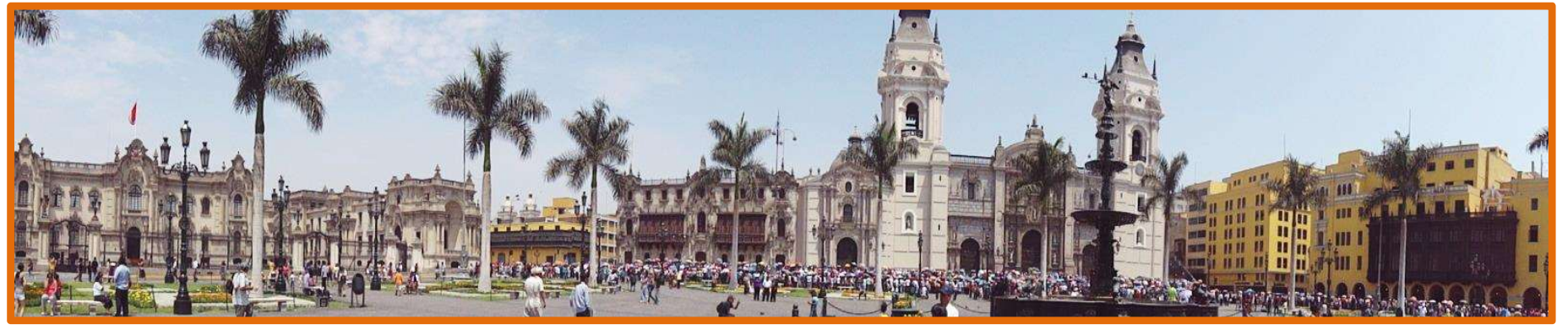

\title{
LATIN AMERICAN CONFERENCE ON PERSON CENTERED MEDICINE
}

\section{From Concept to Action}

\section{Lima, December 18 and 19, 2015 Convention Center of Peruvian Medical College \\ Av. 28 de Julio 776, Miraflores, Lima.}

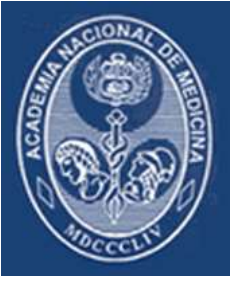

Organized by the Latin American Network of Person Centered Medicine, the Peruvian National Academy of Medicine (ANM), the National University of San Marcos, the Peruvian University Cayetano Heredia, the Catholic

University St. Toribio de Mogrovejo,

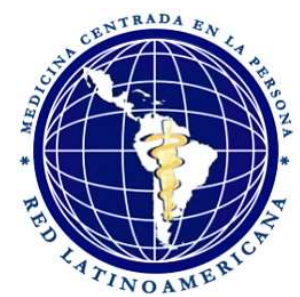
and the National Institute of Health.

Sponsored by the Medical College of Peru, the World Medical Association, the Pan American Health Organization/World Health Organization (PAHO/WHO), National Academies of Medicine of Brazil, Colombia, Chile and Uruguay, and the International College of Person Centered Medicine

MUNISTERIO DE SALUD personas que alendemos personas

I

8 PROGRAM

(8 GALLERY

(7) ABSTRACTS

(7) ACT OF LIMA

( BUENOS AIRES DECLARATION

( ) Literature 
FORM

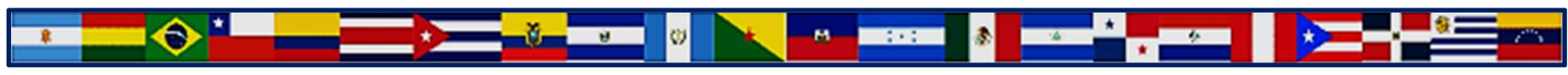

\section{PROGRAM}

\section{First Day: December 18, 2015}

8:30 - 9:00 Registration

9:00-10:00 Opening

Chairs: Oswaldo Zegarra (President, National Academy of Medicine of Peru, ANM Peru), Raúl Morales (Deputy President, ANM Peru), Rodolfo Armas (President, ANM Chile), Juan Mendoza Vega (President, ANM Colombia), Oscar Cluzet (ANM Uruguay), Alberto Perales (Latin American Network of PCM), Miguel Jorge (World Medical Association), Juan Mezizich (International College of Person Centered Medicine).

9:00 - 9:10 Welcome words (Oswaldo Zegarra, Presidente de la ANM Perú)

9:10 - 9:30 Latin American Development and the Clinician-Patient Relationship (Alberto Perales, ANM Perú)

9:30 - 9:50 Institutional Relations towards Person Centered Health (Miguel Jorge, World Medical Association and Brazilian Medical Association)

9:50 - 10:00 Questions and Answers

10:00 - 13:30 Session One: Person Centered Clinical Care

Moderators: Oscar Cluzet (ANM Uruguay), César Cabezas (ANM Perú)

10.00 - 10.20 Person Cenetered Clinical Care Models (Juan Mezzich, ICPCM)

10.20 - 10.40 Clinical Communication (Salomón Zavala, Universidad Nacional Mayor de San Marcos, UNMSM)

10.40 - 11.00 Comprehensive Diagnosis Collaborative Models (Javier Saavedra, Ybeth Luna [Perú]; Elvia Velásquez. [Colombia])

\section{$11.00-11.20 \quad$ Coffee Break}

11.20 - 11.40 Shared Therapeutic Decisions (Juan Limo, Peruvian University Cayetano Heredia, UPCH)

11.40 - 12.00 Colombian Perspectives on Person Centered Clinical Care Juan Mendoza Vega, Presidente ANM Colombia)

12:00 - 12:20 Patient Centered Medicine: A View from Chile (Rodolfo Armas, ANM de Chile)

12:20 - 12:40 Emergencies and Person Centered Medicine (Raúl Morales, ANM Perú)

12.40 - 13.20 Roundtable between speakers and audience

13:20 - 13:30 Conclusions (Raúl Morales, ANM Perú; Alberto Perales, Latin American Network)

13:30 - $15.00 \quad$ Intermission

13.30 - $15.00 \quad$ Executive session organizers: Latin American Network of PCM

15:00 - 18:30 Session Two: Person Centered Health Research

Moderators: (Rodolfo Armas, ANM Chile; José. Pacheco, Academia Peruana de Cirugía)

$15.00-15.20$

$15.20-15.40$

15:40- 16:.00

16.00

$16: 00-16: 40$

$16: 40-17: 00$

17:00- 17:20

$17.20-18.20$

$18: 20-18: 30$
Person Centered Ethics and Health Research (Fredy Canchibuamán, Instituto Nacional de Salud) Research for Person Centered Precision Medicine (Pilar Mazzetti, Former Minister of Health of Peru) Person Centered Health Innovations (Ernesto Gozzer, Jefe del Instituto Nacional de Salud)

\section{Coffee Break (It will be served during the teleconference)}

Person Centered Medicine: Challenges from Ethics to Public Health (Carla Sáenz, PAHO/WHO) (Teleconference from $\mathrm{W}$ ashington $\mathrm{DC}$ )

Person and Community Centered Epidemiological Studies (Javier Saavedra UPCH)

From Scientific Research to Health Policy Design (Eduardo Pretell, Former Minister of Health of Peru)

Roundtable between speakers and audience

Conclusions (Raúl Morales, ANM Perú; Alberto Perales, Latin American Network) 


\section{Second Day: December 19, 2015}

9:00 - 12:30 Session Three: Person Centered Medical Education

Moderators: (Juan Mendoza Vega, President Academia Nacional de Medicina de Colombia; Zuño Burstein, ANM Perú)

9:00 - 9.20 Person Centered University Education (Patricia Campos, Rector, Catholic University St. Toribio de Mogrovejo)

9:20 - 9.40 Person Centered Methods for Educational and Curriculum Structure (Herman Vildózola, Dean Faculty of Medicine, National Major University of San Marcos, UNMSM)

9:40 - $10.00 \quad$ Interdisciplinary Professional Education (Patrick Wagner, ANM Perú)

10:00 - 10.20 Tutoring and Student Professional and Personal Development (Carlos Saavedra L, UNMSM)

10:20 - 10.40 Coffee Break

10.40 - 11.00 Personal, Professional and Pedagogical Development of Professors (Lucía Llosa, Dean Faculty of Medicine, Peruvian University Cayetano Heredia, UPCH)

11.00 - $12.20 \quad$ Roundtable between speakers and audience

12:20 - 12:30 Conclusions (Raúl Morales, ANM Perú; Alberto Perales, Latin American Network)

12:30 - 14:00 Intermission

14:00 - 17:30 Session Four: Person and Community Centered Public Health Moderators: Melitón Arce (Former Deputy Minister of Health of Peru), Ernesto Rios (ANM Perú)

14:00 - 14.20 Person Centered Systems of Integrated Health Care (Oscar Ugarte, Former Minister of Health of Peru)

14:20 - 14.40 Person and Community Centered Integrated Family Health Care (Sofía Cuba, UPCH)

14:40 - $15.00 \quad$ Person and Community Centered Primary Care and Mental Health (Yuri Cutipé, Ministerio de Salud del Perû

15:00 - 15.20 Coffee Break

15:20 - 15:40 Person and Community Centered Public Health (Fernando Carbone, Former Minister of Health of Peru)

15:40 - 16.00 Person and Community Centered Social Determinants of Health (Eugenio Villar, World Health Organization)

16.00 - 17:20 Roundtable between speakers and audience

17:20 - 17:30 Conclusions (Raúl Morales, ANM Perú; Alberto Perales, Latin American Network of PCM)

17:30 - 18-30 Final Session

Chairs: Oswaldo Zegarra, Raúl Morales, Juan Mendoza, Alberto Perales, Juan Mezzich

- Presentation and Adoption of the Lima Declaration

- Next Activities on Person Centered Medicine

- Closing Information: Peruvian National Academy of Medicine Secretary, Telf.: +51-1-652-3819 


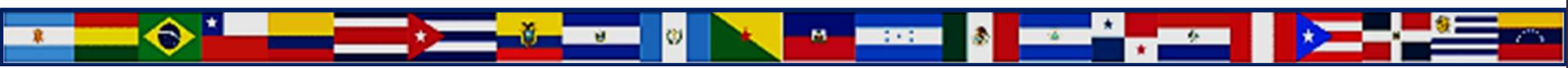

